\title{
Dynamic Modelling of Energy Transitions Using a Coupled Modelling-Narrative Approach
}

\author{
$\underline{\text { E.A. Moallemi }}^{\text {a }}$, F.J. de Haan ${ }^{\text {a,b }}$, B.A. George ${ }^{\text {a,c }}$, J.M. Webb $^{d}$ and L. Aye ${ }^{\text {a }}$ \\ ${ }^{a}$ Renewable Energy and Energy Efficiency Group, Department of Infrastructure Engineering, The University \\ of Melbourne, Victoria, Australia 3010 \\ ${ }^{b}$ Melbourne Sustainable Society Institute, The University of Melbourne, Victoria, Australia 3010 \\ ${ }^{c}$ Integrated Land Water Management Program, International Centre for Agricultural Research in the Dry \\ Areas, Cairo, Egypt \\ ${ }^{d}$ School of Chemistry, The University of Melbourne, Victoria, Australia 3010 \\ Email:emoallemi@student.unimelb.edu.au
}

\begin{abstract}
Energy transitions are a matter of competitions between multiple emerging systems and a dominant, established system. Understanding the complex dynamics of these interactions can assist betterinformed decision making and policy interventions. This paper presents a coupled modelling-narrative approach, consisting of a System Dynamics (SD) model interlinked with a narrative transitions-theoretical framework. The approach is geared at understanding the dynamics of emerging on-grid electricity sources, such as renewables, in power sector transitions.

The value of implementing such a coupled approach is twofold. Firstly, it empowers the SD modelling process. As SD modelling itself is agnostic to the conceptualisation of the (societal) system under study, it is left to the modeller to design an appropriate SD structure - i.e. Causal Loop Diagram. The approach presented in this paper provides a narrative theoretical framework based on the state-of-the-art of Sustainability Transitions literature and a generic SD model (applicable to similar energy transition cases) which directly translates the key concepts and dynamical hypotheses. The theoretical framework enables the creation of highly structured narratives that not only provide a clear overview of the case, but also assist the identification of case specific boundary conditions, parameters, feedback loops and therefore in setting up and validating the SD model. Secondly, the close connection between the narrative theoretical framework and the SD model enables considerable explanatory power that cannot be obtained from simply using a model or a narrative. Where the narrative case description, for example, outlines the developments following a certain policy intervention, the SD model allows interrogating the detailed interactions of the chain of causes and consequences following the intervention. SD models are able to represent and reproduce complex causal relations including feedbacks, non-linearity, threshold effects and time delays - dynamics which are impracticable to analyse with human mental models alone.
\end{abstract}

This paper presents how the SD model is structured based on the core concepts of the narrative theoretical framework. Examples from an existing application by the authors of the framework on the case of the emergence of on-grid solar electricity in India are used to illustrate how the coupling of the SD model with the narrative theory helps addressing questions going beyond modelling or narrative analysis in isolation.

Keywords: $\quad$ Transition modelling, sustainability transition, system dynamics approach, renewable energy 


\section{INTRODUCTION}

Indian electricity sector, as the third largest in the world in terms of power generation, is a complex sociotechnical system. It is composed of both conventional (e.g. coal, diesel and gas) and renewable (e.g. wind, solar and small hydro) sources. It is multi-dimensional, engaged with technical and financial systems (via manufacturing of energy technologies and domestic/foreign investment respectively) and also deeply integrated into the society (via electricity demand and consumption's pattern of users). This complex system has been constantly changing since its beginning, and government has been trying to manage its dynamics for the sake of energy poverty, energy security and emissions reductions. This has made the understanding of this complex dynamics, in a way that how different forms government's interventions can reshape it, a demanding area of research.

Several qualitative and quantitative approaches have been introduced in the body of knowledge in order to understand the dynamics, among them are Transition Studies and System Dynamics (SD) approach. Transition Studies characterises the multi-level and multi-dimensional dynamics of societal system and provides a qualitative and narrative story of change (Geels 2005). SD on the other hand is a modelling approach which explains how reinforcing loops and death spirals (generally known as feedback loops) shape the dynamics of the system (Sterman 2000) in quantative terms. While the complexity of the dynamics has been frequently acknowledged in Transition Studies, they have been unable to grasp non-linearity, time delay and multi-causality of changes with a narrative story and by the mental human mind alone. Considering the strengths and limitations of each approach, the recent developments have favoured a combined quantitativequalitative standpoint or a modelling-narrative approach (Holtz et al. 2015, Robertson 2015). This paper aims to present a combined approach and to propose the structure of a quantitative model supported by qualitative narratives specified for the transition of electricity generation sector towards renewable sources. System Dynamics (SD) approach is employed to represent the structure of the quantitative model, and Transition Studies with the integrated framework, developed by the authors (Moallemi et al. Submitted), is used to specify the qualitative narrative. The approach is geared at understanding the dynamics of emerging on-grid renewable electricity sources in Indian power sector transitions based on the previous studies by authors.

The paper has six sections. After Introduction, Section 2 presents the step-by-step process of the coupled modelling-narrative approach. Section 3 gives a brief overview of the narrative framework. Section 4 develops the high-level structure of SD model based on the narrative theoretical framework. Section 5 presents the offerings of a quantitative model to a qualitative narrative story in the specific case of Indian electricity sector. It discusses how the SD model can assists in comprehending causal relations between market privatisation and the rise of renewables. Finally, Section 6 concludes the paper with the summary of findings.

\section{THE COUPLED MODELLING-NARRATIVE ANALYSIS OF TRANSITION DYNAMICS}

Transition Studies, as an emerging interdisciplinary field, theorises the dynamics of transitions and develops qualitative narratives for societal transformations. System Dynamics approach, as a technique for modelling complex systems, proposes quantitative models for the development of a sector or a technology. The coupled approach developed in this study aims to transcend the individual limitations of modelling and narrative techniques in isolation and to bring an improved understanding from the transformation of societal sectors such as energy sector. The step-by-step process of the coupled modelling-narrative analysis is presented below though it should be noted that the details of the steps are highly dependent on the sector and the context of study.

Step 1 - Description of transition dynamics: the outcome of this step is the qualitative description of transition in terms of initial system's state, conditions, mechanisms and patterns of changes, and new system's state. This is the step when the narrative transitions-theoretical framework ${ }^{1}$ is applied in the case study and the narrative is generated. Three phases are involved in this step. The first is data collection with archival research (i.e. review of government and international organisations' documents) and semi-structured interview with experts as collection methods. The second is data analysis and interpretation of on-the-ground knowledge with coding, categorizing and linking data to the narrative framework as analysis methods. The third is to assess the trustworthiness of outcome with Triangulation technique.

Step 2 - Learning from qualitative narrative: looking back into the qualitative narrative of transition can guide the SD modelling process. Qualitative narrative with its concepts and mechanisms of change can demarcate the system in transition and identify the main components of the dynamics in Causal Loop

\footnotetext{
${ }^{1}$ Shortly referred to as narrative framework or simply the framework from now on.
} 
Diagram. It also specifies the feedback loops between these components as well as actors' interactions as the reasons for time-delay and non-linearity in the dynamics of transition.

Step 3 - Computational modelling of transition: the outcome is a transition model quantitatively exploring the competition between emerging and established systems in a transition. The modelling process starts by developing a dynamics hypothesis and by representing the identified cause-and-effect relations in a Causal Loop Diagram. It is based on the interactions between different actors and feedbacks already identified by the narrative framework in Step 2. The dynamics hypothesis is implemented in System Dynamics software package, i.e. Vensim Professional 6.3, and represented by a Stock and Flow Diagram. The variables of model are quantified with the data collected in Step 1.

Step 4 -Cross analysis of the quantitative and qualitative results: the quantitative output of the model (resulted from simulation runs) is supposed to corroborate the qualitative narrative described in Step 1. The cross analysis of quantitative and qualitative results improve our understanding of transition in different ways: 1. the framework's output can be used for the validation of the modelling results. i.e. whether the simulation results follow the historical transitions, 2. the framework's output also complements SD model by providing an explanation for the governance and cultural influences which are not captured in a SD model. On the other hand, 3. the modelling results give quantity certainty to the narrative output and make them more explicit and tangible, and 4. the quantitative modelling allows inferring the complex behaviour of the system and also predicting its future responses of which qualitative approaches are in capable due to the involved non-linearity and time delays.

\section{AN OVERVIEW OF THE NARRATIVE THEORITICAL FRAMEWORK}

Transition Studies is an interdisciplinary field, from innovation studies (Rogers 1962) and integrated assessment (Rotmans 1998), and are traced back to late 1980s and early 1990s. The field of Transition Studies seeks to understand and also to influence long-term, irreversible and evolutionary processes of change known as transitions. Rooted in two major theories of transition (Geels 2002, de Haan 2010), the narrative framework conceptualises the dynamics of societal transformation, a dynamics shaped by actors' interactions in a partially market condition and with a strong influence of the government's interventions. In the following, a very brief overview of the framework is presented based on an earlier study done by authors (Moallemi et al. Submitted).

The framework conceptualises Indian electricity sector as a Socio-Technical System, composed of conventional and renewable Constellations as competing sub-systems, and surrounded by climate change concerns, global oil price's shock and government policies in External Environment. Constellations (and in general socio-technical system) are functioning normatively; they work in order to satisfy Societal Needs. What resides inside constellations and gives them the power to perform their functioning is System's Actors, e.g. generators and distributors, and their Decisions, e.g. investment decision. Actors' decisions are functions of a Constellations' Attractiveness, the attractiveness which is defined based on the satisfaction of actors' objectives with the constellation's properties (considering actors' perception delay of the real value of properties, the importance of each property for actors and also the minimum acceptable value of constellation's property). This is the way that public and private actors' decisions form the underlying mechanisms in transition.

According to the narrative framework, the transformation of a societal system goes through states of slow and fast dynamics, marked with initial state of the system, destabilisation stage, formation stage and the new state of the system. At the beginning, the system is in equilibrium in the sense that all needs are satisfied. However, it starts to break down as soon as some Destabilisation Forces, whether internal or external to the system, influence the way that societal needs are satisfied (such as oil-price shocks that may interrupt generation of power from conventional sources). At this point, some Formation Forces influence the constellations' attractiveness and push the system towards equilibrium again until the new state of the system emerges (e.g. government subsidies which pushes electricity sector towards renewables).

\section{STRUCTURE OF SYSTEM DYNAMICS MODEL GUIDED BY THE NARRATIVE THEORITICAL FRAMEWORK}

The first step in developing a SD model is to build a Causal Loop Diagram. This diagram represents the high-level structure of SD model and explains the causal relations between model variables, a causality that can be positive (a change in one variable leads to a change in another variable in the same direction) or negative (a change in one variable leads to a change in another variable in the opposite direction) (Pruyt 2013). Causal Loop Diagram also identifies (closed) feedback loops shaped between more than two 
variables; loops that reinforce certain behaviour in the system (positive polarity) or loops that seek towards stability (negative polarity). In this section, we investigate how the narrative framework assists the identification of case specific boundary, feedback loops and therefore in setting up and validating the SD model for the case.

Causal Loop Diagram was developed around the role of societal needs in transition. Based on the description of Indian electricity sector's transition from 1970s to present with the narrative framework (Moallemi et al. 2015), the sector is seeking to satisfy three societal needs: providing the required electricity for household, industry and agriculture (Supply-Demand Balance component), reducing the fuel imports such as coal and oil (Energy Security component) and reducing emissions produced by conventional sources (Environmental component). As long as the needs are satisfied, the electricity system is in the state of equilibrium. The system starts to transform as soon as some driving forces such as a sharp increase in oil price destabilise the conventional-based equilibrium, and some other driving forces such as concerns on the climate change push it towards a renewable dominant state. The framework (Figure 1) describes the dynamics of transition considering how these internal (represented by blue colour) and external driving forces (represented by red colour) in sequential stages of Destabilisation and Formation change the dominancy of different constellations (i.e. conventional and renewable sources) in electricity sector.

Internal driving forces are characterised by four main feedback loops (either destabilising or formative) in Causal Loop Diagram (see Figure 1): Demand Balancing Loop, Generation Scheduling Loop, Investment Loop and Resource Depletion Loop. Demand Balancing Loop explains the goal seeking behaviour of the Demand component. It explains how an initial increase in electricity demand disturbs the balance between supply and demand (a larger demand than supply), raises the price of generated electricity, and subsequently feedbacks with a drop in initial demand (due to the increased price). Generation Scheduling Loop discusses the instant response of electricity generation to a change in the price of electricity in spot market condition, where the hourly generation is adjusted based on peak/off-peak demands. Generators utilise the installed capacity and generate electricity as long as the marginal benefit of generation outweighs its marginal operating cost. The loop starts with an increase in generation and subsequently the improvement of supplydemand balance which leads to a drop in the price of electricity. The lower price reduces the marginal benefits of generators, and results in a decrease in generation at the end. While the impact of Demand Balancing Loop and Generation Scheduling Loop on the dynamics of the electricity system appears in shortterm, Investment Loop and Resource Depletion Loop influence the system in long-term. Investment Loop is a balancing loop, centred around the investment decision based on the expected profits. Accordingly, an increase in the price of generated electricity signals the more profit for the investors in long-term. As financial resources of private investors flow into the power sectors, the acquisition of capital (generators) and installation of capacity expand and the generation of electricity increases. However, the increase in generation and a narrower gap between supply and demand bring down the price of electricity and discourage private sector from further investment. Finally, Resource Depletion Loop represents the limit in electricity generation due to the finite resources. As generation from renewable (or conventional) goes up, the available resources in terms of potential areas for full load hour electricity generation from renewables (or fossil fuel sources for conventional) shrink (with a significant delay). This affects the generation negatively and results in a goal-seeking behaviour for that in long-term.

External forces are mainly the result of Corrective Policy Loops or landscape forces which influence the dynamics of the system, but are not easily affected by the system's behaviour. Corrective Policy Loop deals with the decision of policy makers regarding the type and intensity of energy policies based on the state of societal needs and with an aim to improve the satisfaction of societal needs. Supply-demand component as a societal need is imbalanced when there is a lack of enough investment and the electricity generation does not grow with the same pace with the escalating demand. Electricity generation with high dependency on conventional resources can also result in more energy import and more greenhouse gas (GHG) emissions which adversely affect Energy Security and Environmental components. In response to these negative feedbacks, government devises supportive policies in order to influence the internal dynamics and push the system towards the equilibrium state.

According to the narrative framework, these destabilising and formative driving forces change the dominancy of conventional and renewable constellations in the electricity sector through actors' interactions. Therefore, actors' interactions are placed at the heart of each component in SD model. They are developed based on the investor's decision regarding the share of investment in renewable and conventional sources (in Investment component), distributor's decision regarding the share of renewable and conventional in allocated electricity (in Investment component), provider's decision regarding the volume of capital acquisition and installed capacity (in Capital component), generator's decision regarding the amount generated electricity from 
renewable and conventional sources (in Generation component) and the government's decisions regarding the type and intensity of energy policies in supporting renewables (in Policy component). The inclusion of actors' interactions in SD model is explained with more details in the next two sections.

Having explained the structure of the model, the SD modelling is continued by developing the stock-flow diagram for each model's components considering the (identified) feedback loops among them. The model is implemented in Vensim Professional 6.3. The SD model is going to be used for reproducing the complex dynamics of socio-technical transition from conventional to renewable electricity systems in India. Therefore, the model outputs will be the emergent properties of this dynamics, both technical and non-technical, including the share of investment, installed capacity, generation and consumption from conventional and renewable electricity systems, the state of supply-demand balance, emissions and fuel import as societal needs and the desired level for policy incentives. The simulation runs and quantitative behaviours of output variables are not discussed here as the paper is intended to discuss how modelling and narrative frameworks are linked. However, the explanation of the dynamics in some components is presented in the next two sections with stock-flow diagrams in order to assist comprehending the impact of policy mechanisms on Indian renewable electricity sector.

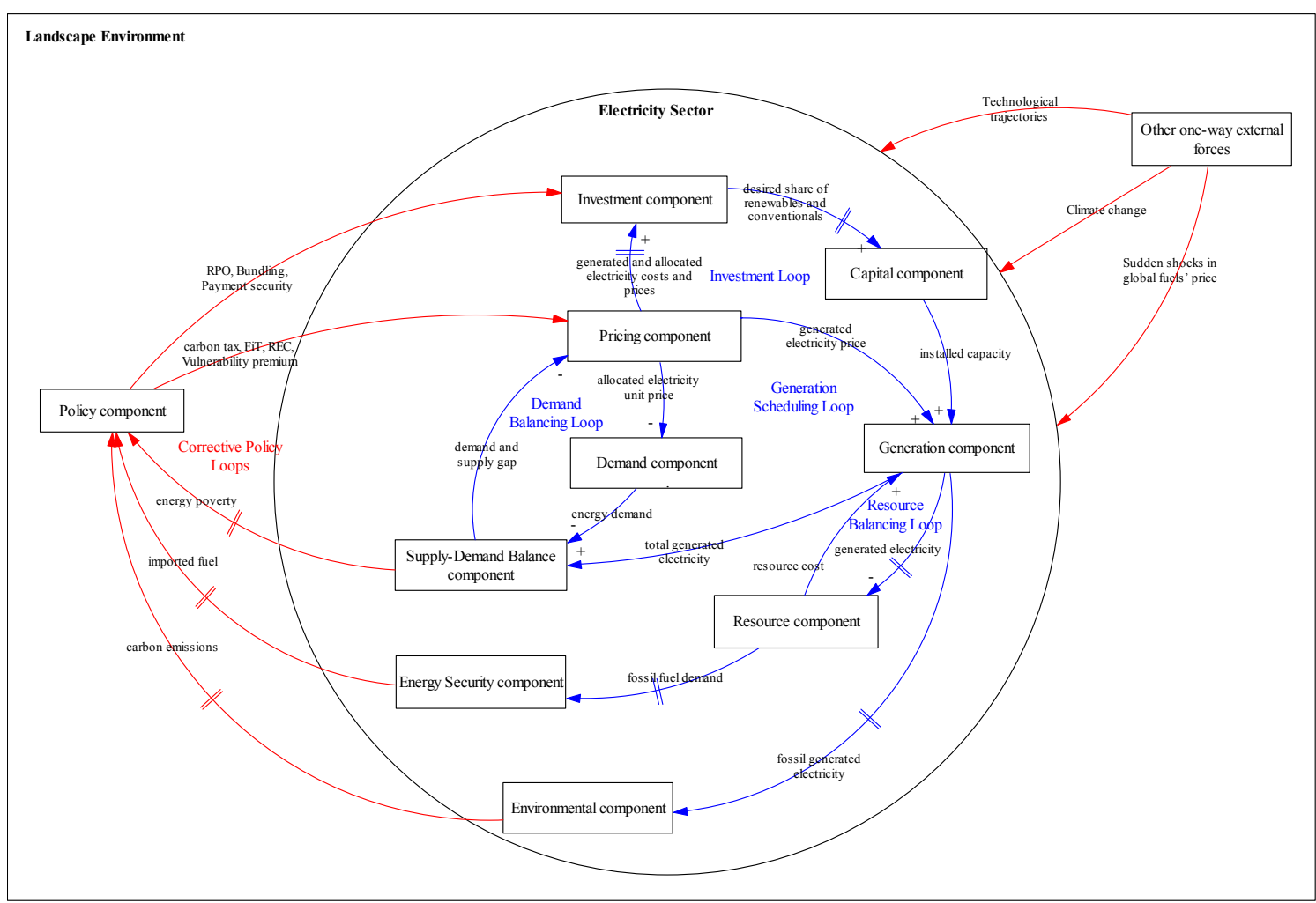

Figure 1. The high-level Causal Loop Diagram of Indian electricity sector transition (arrows show the causal relations and signs on the arrows' heads represent their directional impact).

\section{CAUSAL RELATIONS BETWEEN MARKET PRIVATISATION AND THE RISE OF RENEWABLES}

When the rise of renewables in Indian electricity sector is studied, it is described as highly intertwined with a broader change, a transition from government monopoly to competitive market sector (Moallemi et al. 2015). The electricity sector's enterprises till 1990s were all state-owned. Being highly dependent on the continuous flow of government money, they were working inefficiently. Their inefficient performance aligned with the growing consumption of electricity in the country drained government's budget and interrupted the further expansion of electricity sector. The coincidence of these external and internal destabilising forces damaged the satisfaction of supply-demand balance in early 1990s and invoked government's reaction (with a long delay) to attract investment via market privatisation. The question that is raised here is 'how the market privatisation in 1990s influenced the development of renewables (mostly wind and solar) several years later in Indian electricity sector?' 
The SD model can answer this question by considering stock-flow diagram for model's components and by analysing causal relations, defined in the previous section. Stock-flow diagram is based on the representation of dynamics with some level variables called stock (represented by a rectangle) and some rate variables called flow (represented by a valve). The rate of flow is impacted by auxiliary and/or constant variables, and is defined by an equation explaining their relations. Stocks are the accumulation of flows over time and are determined by the integral of flow's equation over a certain period of time. Running the equation-based model comprising stocks and flows over a certain period of time simulates and reproduces the dynamics of system.

The story of market privatisation can be mainly explained through the interactions in Investment and Corrective Policy Loops in Figure 1. Along with national economic reform in 1991, the government started to amend the Electricity Act 1948 and allowed private investment and 100\% foreign investor ownership to substitute public funds in the electricity sector. As an external force coming from landscape environment, this market privatisation with the attracted private and foreign investments has triggered the total growth of investment in Investment component (see Figure 2). Market privatisation had also another important impact which was the presence of semi-rational and self-interested actors (i.e. investors, generators, distributors) whose decisions were based on the cost-benefit analysis, a decision which could be redirected by government policies towards investment on renewables. Semi-rational decisions of actors in supporting conventional or renewable options are defined as a function of their attractiveness. The attractiveness of each option at each time is equivalent to extend that option's properties satisfy actors' objectives considering actors' perception delay of real values, importance of each property for actors' objectives, and the reference levels. According to Figure 2, the investors' decision to invest is a function of two main properties of electricity options: Rate of Investment (ROI) and payment security. While ROI is dependent on the cost and benefit of renewable generation, payment security is a property which is dependent on the decision of distributors in allocation of electricity demand to different options. The more the purchase from renewables is, the higher the value for payment security is.

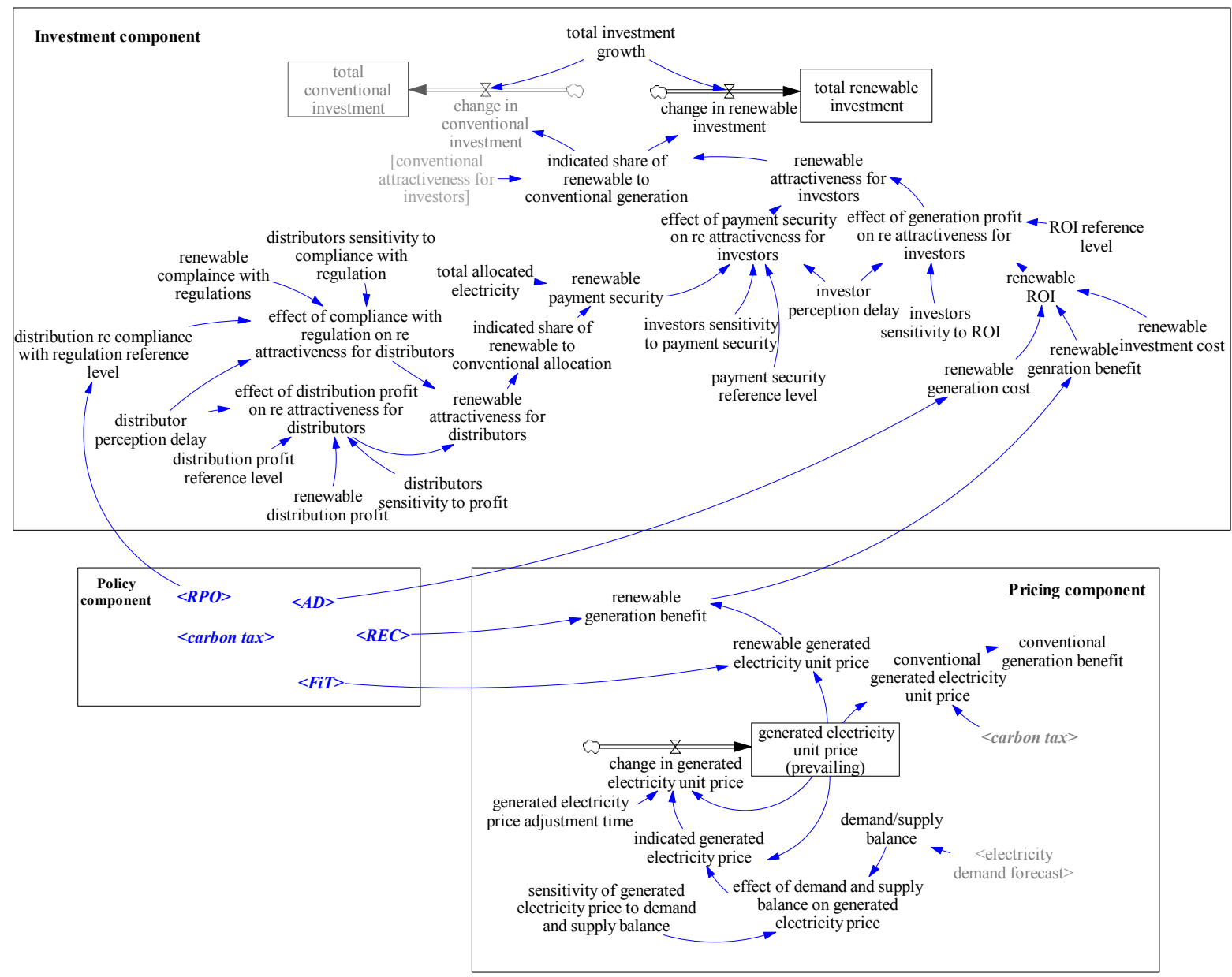

Figure 2. Stock-flow diagram for Investment and Pricing components considering Policy component as external forces. 
Though Investment Loop provided opportunity for further expansion of installed capacities and more generation, rational actors' decisions were initially favouring conventional in 1990s due to their profit attractiveness. However, having more conventional generated electricity was damaging energy security and environmental components and was alerting policy makers for a respond. Indian government started to bring back the system to equilibrium with market-based policies. Market policies, mainly started from Electricity Act 2003, were influencing the properties of electricity options and making them more in favour of renewables. This was the point where Corrective Policy Loop took shape and reoriented Investment Loop towards investment on renewables. On the one hand, policies such as feed-in tariffs (FiTs) and renewable energy credits (RECs) increased the benefit coming from renewable electricity generation via Pricing component and improved renewable's ROI as a decision factor in investors' decision making (see Figure 2). FiT also kept the price of electricity attractive for investors even after the increase of generation which would normally resulted in a drop in price. On the other hand, policies such as renewable purchase obligations (RPO) influenced the reference level for compliance with regulation as an important factor in distributors' decision making. With the increase in the share of renewable to conventional allocated electricity, the payment security for renewables was improved. It elevated the attractiveness of renewables for investors and caused more investments to flow towards renewable installed capacity (Capital component) and then renewable generation during late 1990s and 2000s.

\section{CONCLUSIONS}

SD modelling and narrative frameworks in Transition Studies are both explaining large-scale system's transformations but with different quantitative (model-based) and qualitative (narrative-based) approaches respectively. The integration of modelling and narrative techniques has been recently advocated in the literature in a sense that it can remove the individual limitations of each approach. On the one hand, the narrative transitions-theoretical framework delineates the mechanisms of change involved in SD model and shapes its structure through causal loop and stock-flow diagrams. It also enables explanatory power to the model-produced quantification of the policy's impacts. On the other hand, SD model gives quantity certainty to the narratives and enables them to better explain the underlying reasons behind non-linear causes and consequences of policies.

The integration is important for both descriptive and prescriptive policy studies. In a current case study, the coupled modelling-narrative approach has been applied for investigation of Indian energy transition in order to explain the rise of renewable electricity in Indian power sector. Four causal loops including Demand Balancing Loop, Generation Scheduling Loop, Investment Loop and Resource Balancing Loop were identified. Each of them acts as a virtuous (constructive) or vicious (destructive) loop, pushing forward or hampering the transition towards a renewable-based electricity sector. The interplay between the narrative and the model also clarified the relation between market privatisation and the boost of renewable electricity development.

\section{REFERENCES}

de Haan, F. J. (2010). Towards transition theory. Ph.D. thesis, Erasmus University Rotterdam.

Geels, F. (2002). Technological transitions as evolutionary reconfiguration processes: A multi-level perspective and a case-study. Research Policy 31(8-9): 1257-1274.

Geels, F. (2005). Technological transitions and system innovations: a co-evolutionary and socio-technical analysis. Massachusetts, USA, Edward Elgar Publishing.

Holtz, G., F. Alkemade, F. de Haan, J. Köhler, E. Trutnevyte, T. Luthe, J. Halbe, G. Papachristos, E. Chappin, J. Kwakkel and S. Ruutu (2015). Prospects of modelling societal transitions: Position paper of an emerging community. Environmental Innovation and Societal Transitions(0).

Moallemi, E. A., L. Aye, J. M. Webb, F. J. De Haan and B. A. George (2015). India's on-grid solar power development: past, present and future. Woking paper.

Moallemi, E. A., F. J. De Haan, J. M. Webb, B. A. George and L. Aye (Submitted). Transition Dynamics in State-Stimulated Niche Empowerment: Experiences from Indian Electricity Sector. International Journal of Cleaner Production.

Pruyt, E. (2013). Small System Dynamics Models for Big Issues: Triple Jump towards Real-World Dynamic Complexity. Delft, the Netherlands, TU Delft Library: 324.

Robertson, S. (2015). A longitudinal quantitative-qualitative systems approach to the study of transitions toward a low carbon society. Journal of Cleaner Production(0).

Rogers, E. (1962). Diffusion and Adoption of Innovation: Free Press, New York, USA.

Rotmans, J. (1998). Methods for IA: the challenges and opportunities ahead. Environmental Modeling \& Assessment, 3(3), 55-179.

Sterman, J. (2000). Business dynamics: systems thinking and modeling for a complex world, IrwinMcGraw-Hill. 